\title{
Immunomodulatory therapy of human type 1 diabetes: lessons from the mouse
}

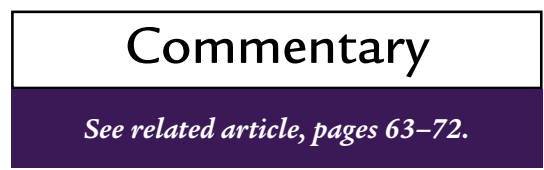

\author{
Jerry P. Palmer \\ Department of Medicine, University of Washington; and Division of Endocrinology and Metabolism, \\ Veterans Affairs Puget Sound Health Care System; Seattle, Washington, USA
}

Address correspondence to: Jerry P. Palmer, Veterans Affairs Puget Sound Health Care System, Endocrinology (III), 1660 South Columbian Way, Seattle, Washington 98108, USA. Phone: (206) 764-2495; Fax: (206) 764-2693; E-mail: jpp@u.washington.edu.

J. Clin. Invest. 108:31-33 (2001). DOI:10.1172/JCI200113445.

In this issue of the JCI, Ryu et al. in the laboratory of Denise Faustman (1) report very stimulating observations on their ability to modulate islet transplant rejection and endogenous $\beta$ cell destruction by autoimmunity in the NOD mouse, a model of human type 1 diabetes. Here, I consider the relevance of their observations to the pathogenesis of type 1 diabetes (T1DM) in humans and, potentially, to immunomodulatory therapy for this disease.

The current report describes multiple experiments in NOD diabetic mice and reaches several major conclusions that may be significant for the human disease (1). The authors found that administering CFA to diabetic NOD mice could prolong the survival of transplanted islets derived from other NOD mice but that this treatment had no such effect when the donor islets were from nondiabetic C57 mice. Hence, CFA blocked islet destruction associated with autoimmunity but not that due to graft rejection, strongly suggesting that these two forms of $\beta$ cell destruction occur through different immunologic mechanisms. The authors attribute the protection afforded by CFA to stimulation of TNF- $\alpha$ production, since the effect could be blocked by administering a TNF- $\alpha$ antibody. Histologic examination of CFA-treated mice transplanted with NOD islets showed that after prolonged normoglycemia but subsequent relapse, both the endogenous pancreatic islets and the transplanted islets contained inflammatory infiltrates. There were no intact islets in the animals' pancreata. Transplantation of islets from a mutant C57 strain that fails to express class I MHC antigens (due to a targeted deletion of the $\beta 2$ microglobulin $[B 2 m]$ gene) had an entirely different effect: After prolonged normoglycemia, CFA-treated mice transplanted with $B 2 m^{-/-}$C57 islets car- ried no intact transplanted islets but their endogenous pancreatic islets were well formed and devoid of lymphocytes. Lymphocytes in these preparations were restricted to the circumference of the islets, a distribution sometimes described as peri-insular insulitis.

To test the relative contribution of endogenous and transplanted islets to glycemic control following transplantation, the authors carried out nephrectomies or pancreatectomies and confirmed that endogenous $\beta$ cells maintained normoglycemia in mice transplanted with $\mathrm{B}^{2 \mathrm{~m}^{-/-}} \mathrm{C} 57$ islets (1). In contrast, mice transplanted with NOD islets and treated with CFA depended on the transplanted islets to maintain normoglycemia. These results further support the idea that different immunologic mechanisms mediate $\beta$ cell loss in transplant rejection and in T1DM autoimmunity.

Crucially, these findings also suggest that normoglycemia after islet transplantation can be due to transplanted islet function or to blockade of the T1DM disease process and resumption of endogenous pancreatic islet insulin production and secretion. Another observation makes this point still more clearly: The authors find that established diabetes can be reversed by treating NOD mice with CFA and C57derived splenocytes (1). Thus, it appears that if the T1DM disease process can be interrupted, at least early after the onset of diabetes, sufficient insulin production can be restored to reverse the animal's diabetes. Whether this reversal is due to recovery of insulin secretion by $\beta$ cells that were only damaged but not destroyed by the T1DM disease process or by regeneration of new $\beta$ cells is unknown.

Ryu et al. (1) also restored near-normal glycemia in NOD mice by implanting immunologically protected (alginate- encapsulated) C57 mouse islets intraperitoneally. They report that this pretreatment greatly increased the success of the CFA/C57 splenocyte treatment in reversing existing diabetes. Hence, it appears that normoglycemia improves $\beta$ cell recovery when immunomodulatory therapy is used to block the type 1 diabetes disease process.

\section{Implications for human T1DM pathogenesis and therapy}

Current immunomodulatory therapy for pancreas and islet transplantation in humans is broad-spectrum and is intended to promote generalized immunosuppression. In the future, it is very likely that immunomodulation will become much more specific and that therapies effective against rejection will be coupled with others that are effective against the T1DM disease process. After successful pancreatic or islet transplantation, if insulin production falls and diabetes recurs, specific tests will be needed to distinguish whether additional treatments should be directed against rejection or against the T1DM disease process.

Since the $\beta$ cell destruction due to rejection and that due to T1DM are mediated by different immunologic mechanisms, it is possible that the immunological mechanisms that initiate the disease process or drive $\beta$ cell destruction also differ among patients with T1DM. For instance, we and others have suggested, based in part on antigenic, metabolic, and genetic differences, that the latent autoimmune diabetes of adults (LADA, or type 1.5 diabetes) may represent a form of autoimmune diabetes that is different from classical childhood onset type 1 diabetes $(2,3)$. Such differences in T1DM subtype may require tailoring immunomodulatory therapies to target the various etiologies. 


\section{Remission of established T1DM}

The current finding that immunomodulatory therapy can reverse established diabetes in the NOD mouse has important implications for human T1DM. The observation is supported by the previous demonstration that a monoclonal antibody to CD3 could also reverse established diabetes in the NOD mouse (4). Although regeneration of new $\beta$ cells may be involved, it is also very likely that successful blockade of the T1DM disease process allows damaged islets and $\beta$ cells to recover and secrete sufficient insulin to correct the diabetes. Interestingly, Strandell et al. $(5,6)$ found that the $\beta$ cell dysfunction in female NOD mice closely correlates with the severity of the islet mononuclear cell infiltration and is accompanied by defective glucose metabolism. Pancreatic islets with insulitis isolated from these mice had deficient glucose-induced insulin release, but when the islets were cultured for 7 days - during which time the islet mononuclear cell infiltrate was depleted - both insulin release and glucose oxidation showed a complete recovery. Moreover, treatment of female NOD mice with monoclonal antibodies directed against infiltrating $\mathrm{T}$ cells markedly reduced the islet inflammatory reaction and restored islet glucose metabolism to normal $(5,6)$.

In human T1DM, there is also more potential for restoration of endogenous insulin secretion and reversal of diabetes than has been assumed. As Mahon et al. have noted (7), patients receiving cyclosporin experience more frequent and longer-duration remissions than control patients, although the risk/benefit ratio of treating patients with newly diagnosed T1DM with cyclosporin may not justify widespread clinical use. In addition, many diabetic individuals show substantial residual $\beta$ cell function, as assessed by their stimulated blood C-peptide level. As a screening test for the Diabetes Control and Complications Trial (DCCT), all T1DM patients received a mixed meal tolerance test to test for residual $\beta$ cell function. Surprisingly, nearly half of adults with T1DM of 1-5 years' duration had C-peptide levels above $0.2 \mathrm{pmol} / \mathrm{ml}$, as did about $10 \%$ of subjects tested 5-15 years after their initial diagnosis (8). Along similar lines, we have noted a dramatic discrepancy between $\beta$ cell function and mass. In animals receiving subdiabetogenic doses of streptozotocin, $\beta$ cell insulin content and insulin secretion could be zero with $50 \%$ of $\beta$ cell mass still remaining (9). These observations, among many others, strongly support the concept that much of the insulin deficiency in patients with established T1DM is due to $\beta$ cell inhibition or damage, rather than the actual depletion of these cells, raising the prospect that sufficient $\beta$ cell mass remains to restore normoglycemia in many patients if the T1DM disease process can be effectively blocked. This blockade of the T1DM disease process might also allow survival of $\beta$ cells newly formed from ductal or stem cells.

\section{Normoglycemia and immunomodulation}

Faustman and colleagues' conclusion that normoglycemia improves the $\beta$ cell recovery when immunomodulatory therapy is used to block the T1DM disease process (1) is in agreement with several other observations, and the principle is currently used clinically. At a mechanistic level, $\beta$ cell stimulation is known to cause increased $\beta$ cell antigen expression and increased cytokineinduced $\beta$ cell cytotoxicity $(10,11)$. The relative metabolic inactivity of $\beta$ cells under normoglycemic conditions, conversely, may help protect these cells by inhibiting these two processes. In the BioBreeding rat, aggressive insulin therapy with frequent hypoglycemia confers protection against diabetes (12), and in the NOD mouse, inhibition of insulin secretion with somatostatin likewise confers protection (13). In humans with the LADA variant of type 1 diabetes, initial inhibition of $\beta$ cell function with diazoxide helps maintain residual $\beta$ cell function (14). Aggressive glycemic control after pancreas and islet transplantation is standard practice, and short-term clinical remissions are more common in patients receiving aggressive glycemic treatment immediately after diagnosis.

Probably the best human data supporting this concept come from the DCCT. Individuals who entered the trial with high residual $\beta$ cell function (stimulated C-peptide levels between 0.2 and $0.5 \mathrm{pmol} / \mathrm{ml}$ ) and who received the intensive therapy to maintain normoglycemia showed a dramatically slower decline in stimulated C-peptide levels than did those receiving conventional treatments (15). Based upon this large amount of concordant data, aggressive glycemic control will surely be part of all immunomodulatory therapies employed in patients with T1DM. It is also important to emphasize that preservation of endogenous $\beta$ cell function is clinically important for patients with type 1 diabetes. In the DCCT, patients with stimulated C-peptide levels above $0.2 \mathrm{pmol} / \mathrm{ml}$ entered the study with hemoglobin A1c levels approximately $1 \%$ better than those with lower C-peptide levels, and they achieved significantly better glycemic control and consequently had less retinopathy. This improved control, as reflected in lower hemoglobin A1c levels, was achieved with a marked reduction in the frequency of hypoglycemia (15). As success in blocking the T1DM disease process in rodents moves to success in humans, the large number of T1DM patients with small amounts of residual $\beta$ cell function will need treatment to achieve the clinical benefits associated with preservation of their residual $\beta$ cell function.

\section{DPT-1 and other clinical trials}

The success in blocking the T1DM disease process in the NOD mouse reported by Ryu et al. (1) adds another intervention to a very long list of interventions that work in this animal model. These treatments were recently summarized in a review by Atkinson and Leiter (16). Although many interventions are successful if employed early in the NOD mouse prior to the onset of overt diabetes, this treatment is one of only a few that is successful after diabetes is established. Diabetes prevention in the animal models has led to pilot studies in humans and more recently to large-scale clinical trials in humans. The two trials farthest along are the European Nicotinamide Diabetes Intervention Trial (ENDIT), being conducted in Europe and Canada, and the Diabetes Prevention Trial-Type 1 (DPT-1), being conducted in the US and Canada. In both of these trials, nondiabetic relatives of patients with type 1 diabetes who are positive for islet cell antibodies (ICAs) are enrolled. ENDIT is testing the effects of nicotinamide as compared with a placebo. All of the subjects have been enrolled, and the results are to be presented in 2003. DPT-1 includes two independent trials in two separate risk cohorts, one testing parenteral insulin in high-risk individuals, and the other testing oral insulin in intermediate-risk 
individuals. Enrollment for the oral insulin trial is still ongoing. The results of the parenteral study, announced at the American Diabetes Association meeting in June 2001, showed that there was no difference in the rate of diabetes development in those high-risk subjects treated with parenteral insulin as compared with controls.

Although disappointing and surprising, given the ability of parenteral insulin to prevent diabetes in the animal models and in small human pilot trials (17), this DPT- 1 result emphasizes the necessity for basing treatment decisions in humans on properly designed and executed human trials. It is important not to overinterpret these negative findings, since they only apply to high-risk subjects, as defined by the DPT-1, and to insulin at the doses and by the routes tested. The much higher doses of insulin commonly used in the NOD mouse to prevent diabetes and insulin administered by other routes, such as oral or nasal, may still prove efficacious, even for high-risk individuals.

The NIH is committed to a large clinical trials program across the US with the task of testing the efficacy of immunomodulatory therapy against the human type 1 diabetes disease process. This program, termed Type 1 Diabetes TrialNet, will consist of up to 20 clinical centers, core laboratories, a coordinating center, and a data management center and will oversee and conduct immunomodulatory intervention trials for type 1 diabetes in the US. Several interventions in different populations are currently being evaluated with plans for the program to become active in the fall of 2001.

The wide availability of antibody tests such as islet cell antibodies and autoantibodies against glutamic acid decarboxylase (GAD), insulin, and IA2 as indicators of the autoimmune nature of type 1 diabetes has expanded our definition of this disease. In addition to classical childhood-onset type 1 diabetes, $5-25 \%$ of patients with phenotypic type 2 diabetes are positive for one or more of these autoantibodies. ICAs and GAD antibodies are particularly common, and phenotypic type 2 patients positive for either or both of these antibodies have a much more rapid decline in $\beta$ cell function after diagnosis than do antibody-negative patients and consistently require insulin treatment earlier (18). A recent Japanese pilot study on ICA-positive type 2 patients, comparing sulfonylurea with insulin (19), and a recently completed larger study in GAD antibodypositive type 2 patients, also from Japan, showed better preservation of $\beta$ cell function with insulin (20). Unfortunately, the DPT-1 did not confirm the beneficial effect of parenteral insulin on the type 1 diabetes disease process. The divergent findings could be explained by any of several variables, including disease severity at the time of recruitment to the study (established diabetes in one case, as opposed to high-risk status), age, disease subtype (LADA or classical type 1 diabetes), and ethnic background (Japanese or North American). Nonetheless, the importance of this patient population with phenotypic type 2 diabetes but the antibodies of type 1 diabetes needs to be emphasized. Because of the high prevalence of type 2 diabetes, even if only $5-10 \%$ are antibody-positive, this represents a population of patients severalfold larger than the population of patients with classical childhood type 1 diabetes. Effective immunomodulatory therapy that prevents diabetes or preserves residual $\beta$ cell function in patients with autoimmune diabetes will be an important development.

These are exciting times for the fields of autoimmunity and type 1 diabetes. A major challenge is how to translate into clinical medicine the latest findings from basic research at the cellular and molecular levels and from research in animal models. This translation process has resulted in a dramatic improvement in the success of islet transplantation (21). Although parenteral insulin as administered in the DPT-1 did not block the type 1 diabetes disease process, future clinical trials under the auspices of TrialNet, the Immune Tolerance Network, and other organizations will continue this quest until safe and effective immunomodulatory therapy for human type 1 diabetes is found.

1. Ryu, S., Kodama, S., Ryu, K., Schoenfeld, D.A., and Faustman, D.L. 2001. Reversal of established autoimmune diabetes by restoration of endogenous $\beta$ cell function. J. Clin. Invest. 108:63-72.

2. Tuomi, T., et al. 1999. Clinical and genetic characteristics of type 2 diabetes with and without GAD antibodies. Diabetes. 48:150-157.

3. Juneja, R., and Palmer, J.P. 1999. Type 1 1/2 diabetes: myth or reality? Autoimmunity. 29:65-83.
4. Chatenoud, L., Primo, J., and Bach, J.-F. 1997. CD3 antibody-induced dominant self tolerance in overtly diabetic NOD mice. J. Immunol. 158:2947-2954.

5. Strandell, E., Eizirik, D.L., and Sandler, S. 1990. Reversal of $\beta$-cell suppression in vitro in pancreatic islets isolated from nonobese diabetic mice during the phase preceding insulin-dependent diabetes mellitus. J. Clin. Invest. 85:1944-1950. 6. Strandell, E., Sandler, S., Boitard, C., and Eizirik, D.L. 1992. Role of infiltrating T-cells for impaired glucose metabolism in pancreatic islets isolated from non-obese mice. Diabetologia. 35:924-931.

7. Mahon, J.L., Dupre, J., and Stiller, C.R. 1993. Lessons learned from use of cyclosporine for insulin-dependent diabetes mellitus: the case for immunotherapy for insulin-dependent diabetics having residual insulin secretion. Ann. NY Acad. Sci. 696:351-363.

8. 1987. Effects of age, duration and treatment of insulin-dependent diabetes mellitus on residual $\beta$-cell function: observations during eligibility testing for the Diabetes Control and Complications Trial (DCCT). The DCCT Research Group J. Clin. Endocrinol. Metab. 65:30-36.

9. McCulloch, D.K., Koerker, D.J., Kahn, S.E., Bonner-Weir, S., and Palmer, J.P. 1991. Correlations of in vivo beta cell function tests with beta cell mass and pancreatic insulin content in streptozocinadministered baboons. Diabetes. 40:673-679.

10. Hao, W., Li, L., Mehta, V., Lernmark, A., and Palmer, J.P. 1994. The functional state of the beta cell affects expression of both forms of glutamic acid decarboxylase. Pancreas. 9:558-562.

11. Mehta, V., Hao, W., Brooks-Worrell, B.M., and Palmer, J.P. 1993. The functional state of the beta cell modulates IL-1 and TNF induced cytotoxicity. Lymphokine Cytokine Res. 12:255-259.

12. Gotfredsen, C.F., Buschard, K., and Frandsen, E.K. 1985. Reduction of diabetes incidence of BB Wistar rats by early prophylactic insulin treatment of diabetes-prone animals. Diabetologia. 28:933-935.

13. Bowman, M.A., et al. 1996. Immunological and metabolic effects of prophylactic insulin therapy in the NOD-scid/scid adoptive transfer model of IDDM. Diabetes. 45:205-208.

14. Bjork, E., et al. 1996. Diazoxide treatment at onset preserves residual insulin secretion in adults with autoimmune diabetes. Diabetes. 45:1427-1430.

15. 1998. Effect of intensive therapy on residual $\beta$ cell function in patients with type 1 diabetes in the diabetes control and complications trial: a randomized, controlled trial. The Diabetes Control and Complications Trial Research Group. Ann. Intern. Med. 128:517-523.

16. Atkinson, M.A., and Leiter, E.H. 1999. The NOD mouse model of type 1 diabetes: as good as it gets? Nat. Med. 5:601-604.

17. Keller, R.J., Eisenbarth, G.S., and Jackson, R.A. 1993. Insulin prophylaxis in individuals at high risk of type 1 diabetes. Lancet. 341:927-928.

18. Turner, R., et al. 1997. UKPDS 25: autoantibodies to islet-cell cytoplasm and glutamic acid decarboxylase for prediction of insulin requirement in type 2 diabetes. UK Prospective Diabetes Study Group. Lancet. 350:1288-1293.

19. Kobayashi, T., Nakanishi, K., Murase, T., and Kosaka, K. 1996. Small doses of subcutaneousinsulin as a strategy for preventing slowly progressive $\beta$-cell failure in islet cell antibody-positive patients with clinical features of NIDDM. Diabetes. 45:622-626.

20. Kobayashi, T., et al. 2001. Multicenter prevention trial of slowly progressive IDDM with small dose of insulin (the Tokyo Study). Diabetes Metab. Res. Rev. 17(Suppl. 1):S29. (Abstr.)

21. Shapiro, A.M.J., et al. 2000. Islet transplantation in seven patients with type 1 diabetes mellitus using a glucocorticoid-free immunosuppressive regimen. N. Engl. J. Med. 343:230-238. 